\title{
Glucose-regulated protein 94 deficiency induces squamous cell metaplasia and suppresses PTEN-null driven endometrial epithelial tumor development
}

\author{
Jieli Shen ${ }^{1}$, Lijing Yao${ }^{1}$, Yvonne G. Lin², Francesco J. DeMayo ${ }^{3}$, John P. Lydon ${ }^{3}$, Louis \\ Dubeau ${ }^{4}$, Amy S. Lee ${ }^{1}$ \\ ${ }^{1}$ Department of Biochemistry and Molecular Biology, USC Norris Comprehensive Cancer Center, Keck School of Medicine, \\ University of Southern California, Los Angeles, CA 90089, USA \\ ${ }^{2}$ Division of Gynecologic Oncology, Department of Obstetrics-Gynecology, USC Norris Comprehensive Cancer Center, Keck \\ School of Medicine, University of Southern California, Los Angeles, CA 90089, USA \\ ${ }^{3}$ Department of Molecular and Cellular Biology, Baylor College of Medicine, Houston, TX 77030, USA \\ ${ }^{4}$ Department of Pathology, USC Norris Comprehensive Cancer Center, Keck School of Medicine, University of Southern \\ California, Los Angeles, CA 90089, USA
}

Correspondence to: Amy S. Lee, e-mail: amylee@usc.edu

Keywords: endometrial cancer, PTEN, glucose-regulated protein 94 (GRP94), squamous cell metaplasia, $\beta$-catenin

Received: December 23, $2015 \quad$ Accepted: January 30, $2016 \quad$ Published: February 17, 2016

\section{ABSTRACT}

Endometrial carcinoma is the most prevalent gynecologic cancer in the United States. The tumor suppressor gene Pten (phosphatase and tensin homolog) is commonly mutated in the more common type 1 (endometrioid) subtype. The glucose-regulated protein 94 (GRP94) is emerging as a novel regulator for cancer development. Here we report that expression profiles from the Cancer Genome Atlas (TCGA) showed significantly increased Grp94 mRNA levels in endometrial tumor versus normal tissues, correlating with highly elevated GRP94 protein expression in patient samples and the requirement of GRP94 for maintaining viability of human endometrioid adenocarcinoma (EAC) cell lines. Through generation of uterus-specific knockout mouse models with deletion of Grp94 alone $\left(c 94^{f / f}\right)$ or in combination with Pten $\left(c P^{f / f} 94^{f / f}\right)$, we discovered that $c 94^{f / f}$ uteri induced squamous cell metaplasia (SCM) and reduced active nuclear $\beta$-catenin. The $c P^{f / f} 94^{f / f}$ uteri showed accelerated SCM and suppression of PTEN-null driven EAC, with reduced cellular proliferation, attenuated $\beta$-catenin signaling and decreased AKT/S6 activation in the SCM. In contrast to single PTEN knockout uteri $\left(c P^{f / f}\right), c P^{f / f} 94^{f / f}$ uteri showed no decrease in E-cadherin level and no invasive lesion. Collectively, our study implies that GRP94 downregulation induces SCM in EAC and suppresses AKT/S6 signaling, providing a novel mechanism for suppressing EAC progression.

\section{INTRODUCTION}

Endometrioid adenocarcinoma (EAC), the most common gynecological malignancy in the United States, arises from the inner lining of the uterus [1]. EAC has been categorized into two distinct types. Type 1 tumors exhibit endometrioid differentiation and account for approximately $85 \%$ of the cases, whereas type 2 tumors, non-endometrioid, often display serous or clear cell histology [2]. The most common mutation in type 1 tumors is in the Pten tumor suppressor gene which is altered in 40 to $80 \%$ of cases [2]. An endometrial cancer mouse model mimicking human EAC has been established using the progesterone receptor promoter driven Crerecombinase $(\mathrm{PR}$-Cre $)$ to conditionally knockout Pten in the endometrium, resulting in rapid onset of EAC at 4 weeks of age, with infiltration into the myometrium by 8 weeks [3]. Thus, this model is useful for studying the key determinants of EAC development and invasion.

Glucose-regulated protein 94 (GRP94) encoded in humans by HSP $90 B 1$, is a member of the HSP90 family sharing 50\% homology to cytosolic paralog HSP90 [4, 5]. It is a multifunctional protein playing important roles as a molecular chaperone and a $\mathrm{Ca}^{2+}$-binding protein in the 
endoplasmic reticulum (ER) [6, 7]. Homozygous knockout of GRP94 results in embryonic lethality $[8,9]$. As an ER chaperone, GRP94 controls the maturation and secretion of insulin growth factors (IGFs) and the processing of Toll-like receptors [7, 10]. Recently, the Wnt co-receptor, LRP6, has been identified as a new client of GRP94 [11]. When GRP94 was conditionally knocked out in the gut, LRP6 failed to export to the cell surface, and nuclear translocation of $\beta$-catenin was compromised [11]. As the $\beta$-catenin pathway is a key regulator of development as well as tumorigenesis, this suggests that loss of GRP94 could impact cellular differentiation and cancer progression. Invasive cancer requires remodeling of the cell adhesion molecules that maintain cell-cell contacts and intercellular junctions [12]. Another major function attributed to GRP94 is in maintaining cell matrix integrity due to its chaperoning activities for key cell surface adhesion proteins such as E-cadherin and integrins [13-15].

GRP94 is commonly elevated in human cancers [5]. The Grp94 promoter was strongly activated in spontaneous and chemically-induced tumors in mice [16]. GRP94 loss in B cells attenuated multiple myeloma, and GRP94 deficiency in macrophages reduced colitis and inflammation-associated colon tumorigenesis [17, 18]. However, in the bone marrow and hepatocytes, GRP94 loss perturbed extracellular matrix proteins, oncogenic signaling pathways and led to hyper-proliferation of progenitor/stem cells $[13,14]$. Interestingly, repopulation of GRP94-positive hepatocytes was detected in GRP94-deficient livers, correlating with spontaneous hepatocellular carcinoma development in aged mice and promotion of chemically-induced hepatocellular carcinogenesis $[19,20]$. Therefore, GRP94 could have a multifaceted effect on tumorigenesis that is context- and age-dependent. In this study, we report that GRP94 expression is significantly elevated in human endometrial cancer and required for human endometrial cancer cell viability. We further report the creation of two endometrial-specific knockout mouse models with deletion of Grp94 alone or in combination with Pten deletion. Our study reveals that loss of GRP94 in mouse endometrium induces squamous cell metaplasia (SCM), attenuated PTEN-deficiency mediated $\beta$-catenin and AKT/S6 activation in the SCM and suppressed EAC.

\section{RESULTS}

\section{GRP94 mRNA and protein levels are elevated in human EAC and contribute to cell viability}

First, we performed Grp94 mRNA expression analyses using 24 normal and 177 endometrial cancer expression profiles from TCGA [21]. We observed significantly increased Grp94 mRNA expression in tumor versus normal samples ( $p$ value $=4.7 \mathrm{E}-14$ ), and comparable Grp94 mRNA levels in different grade and stage of endometrial cancer (Figures 1A-1C). As many Pten mutations result in decreased expression [2, 22], Pten mRNA is downregulated in tumor samples compared to normal samples $(p$ value $=5.6 \mathrm{E}-16)$ (Figure 1D). Furthermore, examination of GRP94 protein expression in human uterine tissue samples by immunohistochemistry (IHC) showed basal GRP94 expression in epithelial cells in the normal endometrium, and upon development of EAC which is populated by epithelial cells, strong GRP94 expression was detected uniformly in Grade 1, 2 and 3 tumors (Figure $1 \mathrm{E}$ ), in agreement with the RNA-seq dataset analysis. Furthermore, consistent with GRP94 over-expression in endometrial cancer which is predominantly mutated in Pten, in RNA-seq dataset analysis, an inverse correlation was detected between Grp94 and Pten mRNA levels using both normal (green) and tumor samples (red) $(p$ value $=0.001)$ due to the opposite expression changes between Pten and Grp94 mRNA levels from normal to tumor (Figure 1F).

To test the effect of GRP94 deficiency on the viability of human endometrial cancer cells, two cell lines (AN3CA and ECC-1), both reported to have loss of PTEN [23], were examined. Treatment with siRNA targeting Grp 94 reduced its protein level in AN3CA cells by $70 \%$ and in ECC-1 cells by $90 \%$ compared to control siRNA (siCtrl), corresponding with a $35 \%$ and $60 \%$ decrease in viability of AN3CA and ECC-1 cells, respectively, compared to siCtrl, as measured by the WST-1 assay (Figures 1G and 1H). Thus, GRP94 contributes to human endometrial cancer cell viability.

\section{Endometrial GRP94 ablation leads to spontaneous squamous cell metaplasia and loss of active nuclear $\beta$-catenin expression}

To study the role of GRP94 in endometrial cancer in vivo, we utilized genetically engineered mouse models. First, we determined the effect of GRP94 ablation in the normal mouse endometrium. Deletion of GRP94 in the post-natal mouse endometrium was achieved through creation of mice bearing a PR-Cre transgene and Grp94 floxed alleles. The PR promoter is activated in endometrial tissues around post-natal day 3 leading to gene ablation in luminal and glandular epithelial cells at first and subsequently in endometrial stroma and myometrium [24]. The PR-Cre; Grp $94^{f / f}$ mice, referred to below as $c 94^{f / f}$, were analyzed in parallel with littermates lacking $P R$-Cre serving as WT controls. The genotypes of the mice were determined by PCR of tail genomic DNA (Figure 2A). At both 4 and 8 weeks, the $c 94^{f / f}$ uteri were smaller (Figure 2B) and lighter (Figure 2C). Histological examination of uterine sections stained with H\&E revealed two major differences. First, the c94 fff uteri exhibited reduced number of endometrial glands compared to WT (Figure 2D, white arrows), resulting in a flat luminal surface. Second, while the WT uteri were lined with a single layer of columnar cells (black bars), at 8 weeks the $c 94^{\mathrm{f} / \mathrm{f}}$ uteri were lined by multi-layered squamous epithelium (Figure 2D, red bars). In contrast, 
uteri deficient of another ER chaperone GRP78 did not exhibit SCM and were lined with a single layer of columnar cells (Supplementary Figure S1). As demonstrated by IHC, GRP94 expression was largely depleted in the $c 94^{\text {ff }}$ uteri except in occasional stromal cells (Figure 2D).

To confirm that the multi-layered epithelium in $c 94^{f / f}$ uteri represented squamous metaplasia, the expression of p63 and cytokeratin 14 (K14), representing early and late markers of squamous differentiation, respectively, was examined by IHC $[25,26]$. At 4 weeks, p63 expression was detected in some $c 94^{\mathrm{ff} f}$ endometrial epithelial cells located within the luminal lining, indicating initiation of SCM; by 8 weeks $c 94^{f / f}$ endometrial epithelial cells showed robust p63 and K14 expression, confirming their squamous nature (Figure 3A). As expected, both markers were absent from WT endometrium.
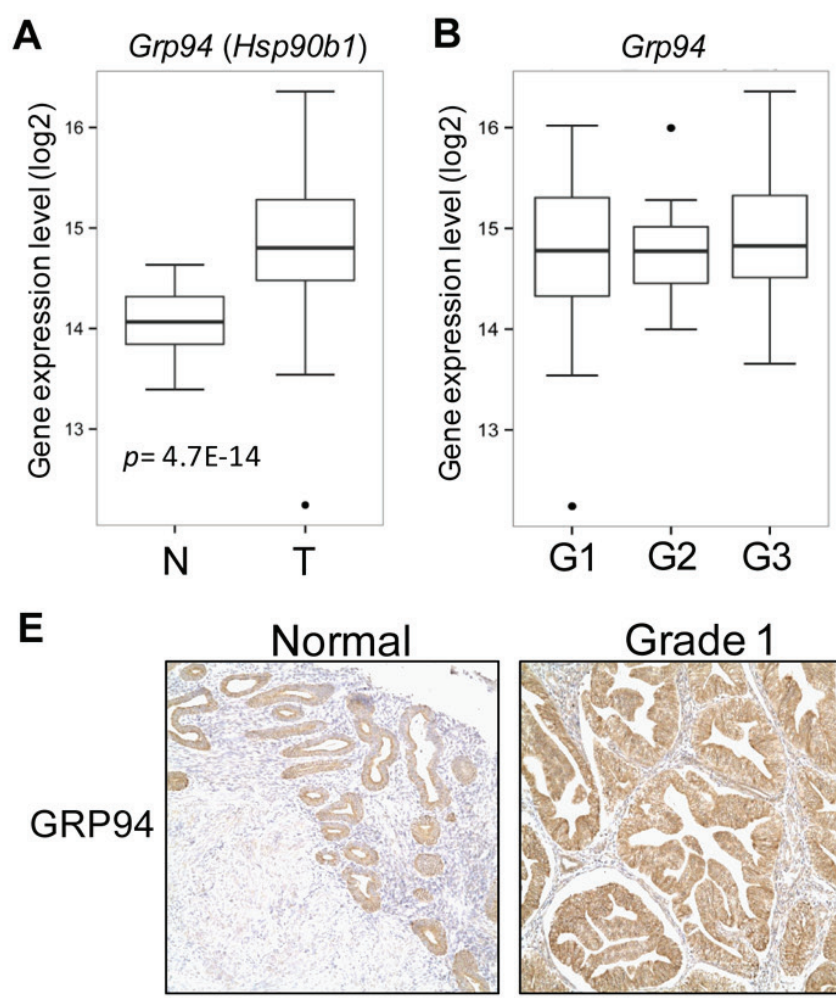

$\mathbf{F}$

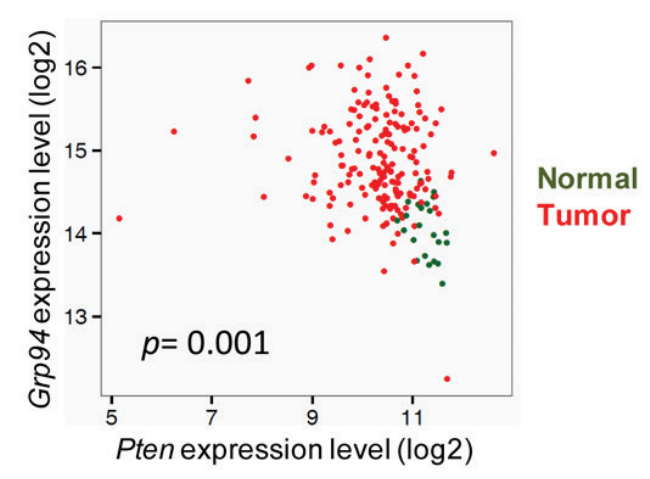

E

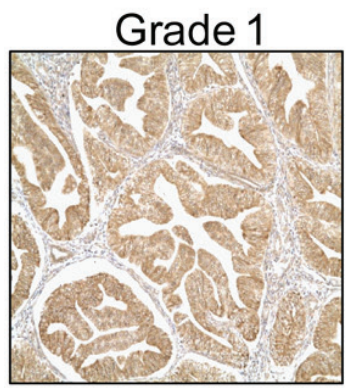

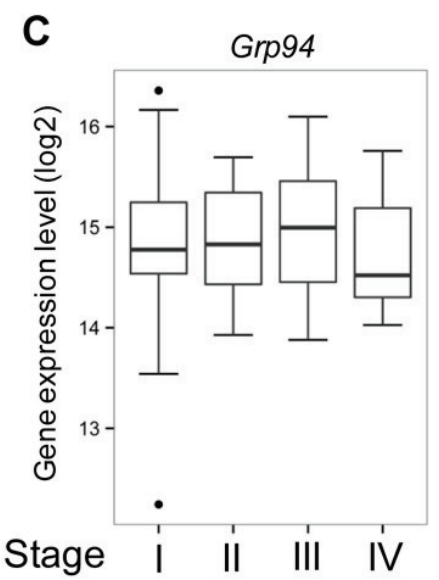

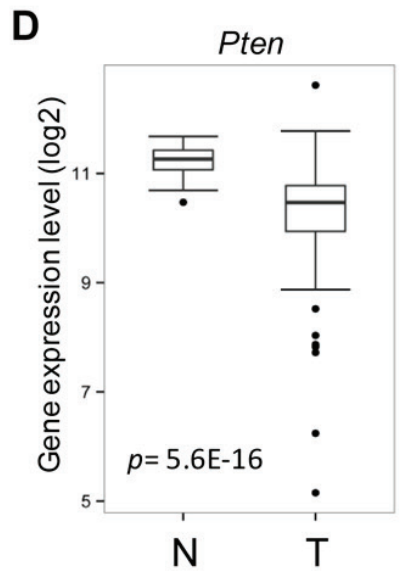

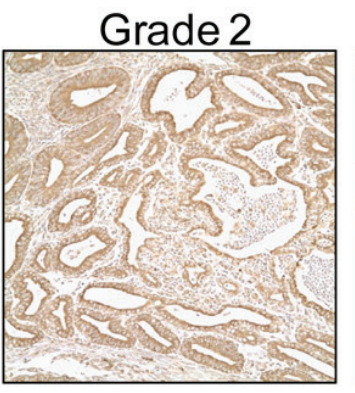

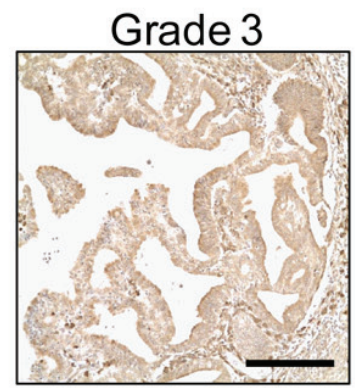

G

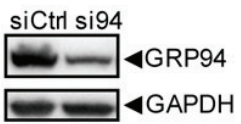

$1.0 \quad 0.3$

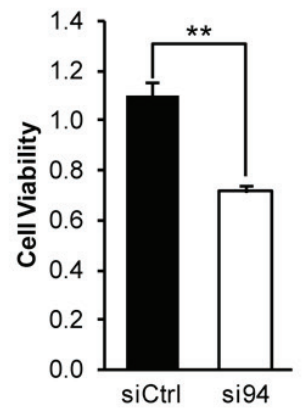

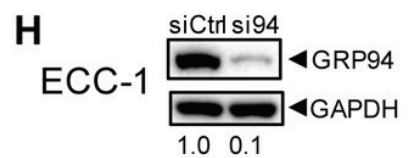

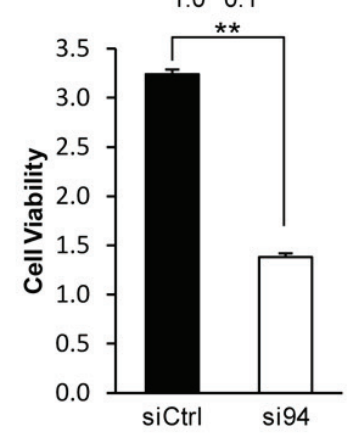

Figure 1: Increased GRP94 expression in human EAC and its effect on EAC cell viability. Grp 94 mRNA expression in A. normal uterine $(\mathrm{n}=24)$ and EAC tissues $(\mathrm{n}=177)$, B. different grade EAC, and C. different stage EAC. D. Pten mRNA expression in normal uterine and EAC tissues. Student's $t$-test $p$ values are indicated. E. Immunohistochemistry (IHC) analysis of GRP94 in human normal uterine and EAC tissues. Scale bar, $50 \mu \mathrm{m}$. F. Scatterplot of Grp94 and Pten mRNA expression of human normal uterine (green) and tumor samples (red). Pearson correlation $p$ value is indicated. Western blot analysis of GRP94 knockdown efficiency in G. AN3CA cells and H. ECC-1 cells. The level of GRP94 reduction after normalization to GAPDH which served as loading control is shown below. WST-1 assay measuring cell viability in si-control (siCtrl) and si-Grp94 (si94) treated G. AN3CA cells at day 4 and $\mathbf{H}$. ECC-1 cells at day 5. Data are presented as mean \pm s.e., ${ }^{* *} p<0.01$ (Student's $t$-test). 
We next tested whether SCM observed in the $c 94^{f / f}$ endometrium could be due to $\beta$-catenin dysfunction since ablation of endometrial $\beta$-catenin has been reported to induce SCM [27, 28]. IHC analysis revealed that at 4 weeks both $c 94^{\mathrm{fff}}$ and WT endometria showed strong active nuclear $\beta$-catenin expression in the columnar epithelium (Figure 3B, box), indicating that GRP94 is not required for $\beta$-catenin activation and nuclear translocation in columnar cells. However, 8 week old $c 94^{f / f}$ endometrium, which had undergone SCM, showed minimal active nuclear $\beta$-catenin expression compared to WT (Figure 3B, box). Total $\beta$-catenin levels were similar in $c 94^{f / f}$ and WT endometria at 4 and 8 weeks (Figure 2B). No spontaneous endometrial cancer was observed in the $c 94^{f / f}$ uteri for at least 9 months (Supplementary Figure S2).

\section{Endometrial GRP94 deficiency suppresses PTEN-null driven adenocarcinoma}

To test the role of GRP94 in type 1 endometrial cancer, which can be induced by PTEN depletion, $P R$ Cre;Pten floxflox $\left(c P^{f / f}\right)$ mice were crossed with Pten ${ }^{f / f} 94^{f f f}$ mice to generate a biallelic deletion strain lacking both
Pten and Grp94 $\left(c P^{f / f 94^{f / f}}\right)$. Littermates lacking the PRCre transgene were used as WT controls. The mouse genotypes were determined by PCR of tail genomic DNA (Figure 4A). At 4 weeks, the $c P^{f f f}$ uteri were considerably larger than the WT and $c P^{f / f} 94^{f / f}$ uteri (Figure 4B), and the average weight of $c P^{f / f}$ uteri normalized against body weight was 5.5 and 2.0 times that of the WT and $c P^{f / f} 94^{f / f}$ uteri, respectively (Figure 4C). Western blot analysis of uterine tissue lysates confirmed minimal expression of PTEN and GRP94 in the respective knockout mice, with GAPDH serving as loading control (Figure 4D).

Histological examination showed that at 4 weeks all $9 c P^{f / f}$ uteri examined contained extensive and aggressive EAC which, in 8 of the 9 cases, replaced more than $70 \%$ of the endometrium (Figure 4E). One out of $7 c P^{f / f} 94^{f / f}$ uteri showed no evidence of malignancy. The remaining 6 $c P^{f / f} 94^{f / f}$ uteri showed a mixture of normal and transformed glands (Figure 4E, white and red arrows, respectively). Five out of 6 showed $30 \%$ or less of the endometrium replaced by tumor, while only one uterus showed more extensive tumor involvement. In addition, all tumors seen in $c P^{f / f} 94^{f / f}$ uteri were separated from the myometrium by a layer of normal endometrium, as opposed to the tumors
A

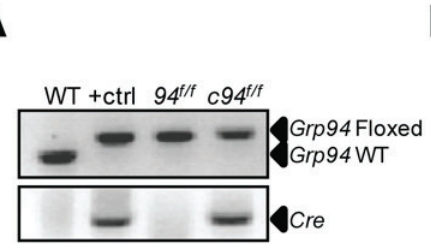

D
B

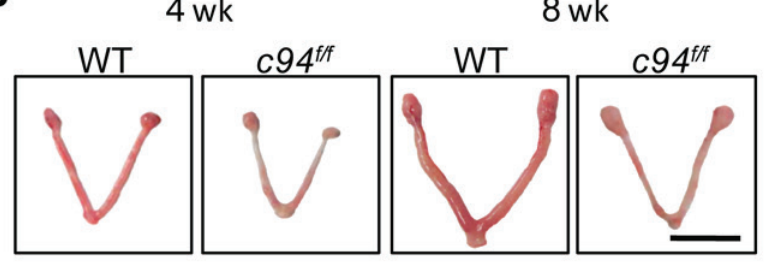

C

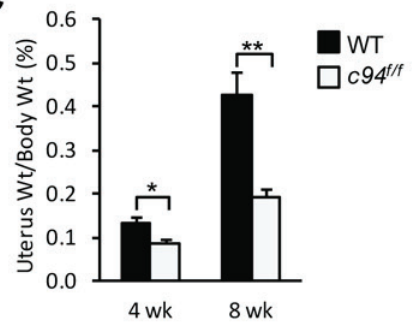

4 wk
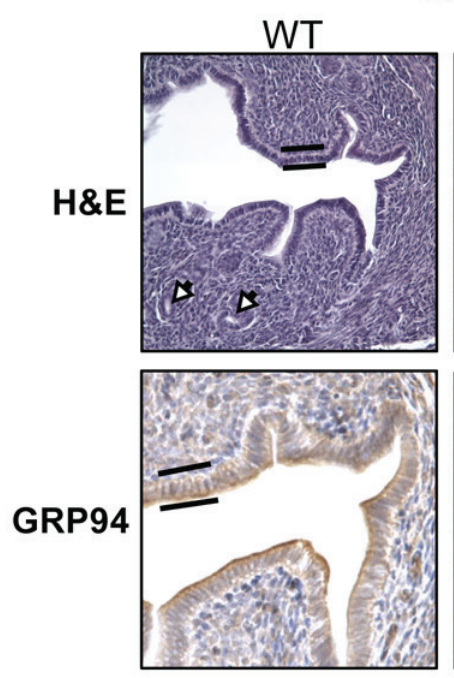

wk
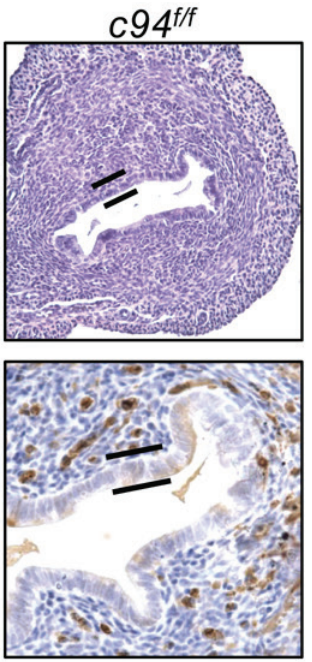

$8 w k$
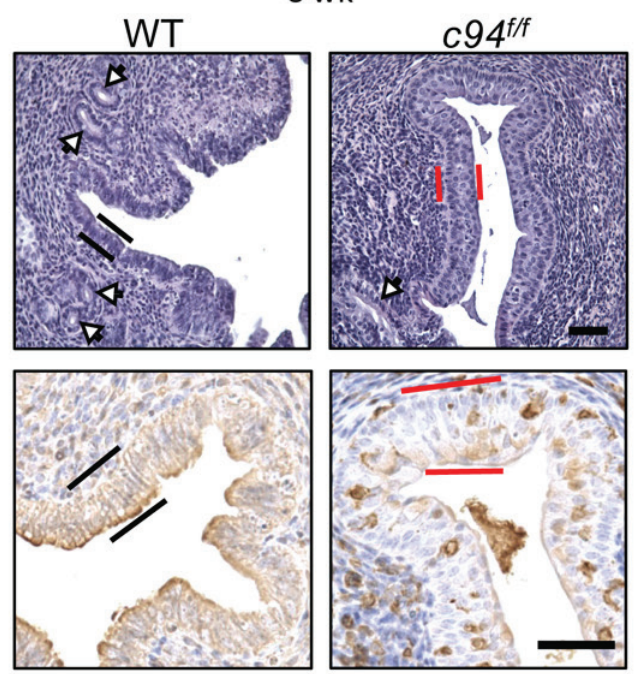

Figure 2: Generation of PR-Cre-mediated GRP94 knockout mouse models. A. Representative mouse tail PCR genotyping of the indicated alleles. B. Representative gross anatomy of uteri of the indicated genotypes at 4 and 8 weeks. Scale bar, $1 \mathrm{~cm}$. C. The ratio of uterine weight to body weight in WT and $c 94^{f / f}$ mice at 4 weeks ( $\mathrm{n}=16$ and 5 , respectively) and 8 weeks ( $\mathrm{n}=4$ and 11 , respectively). The data are presented as mean \pm standard error (s.e.), ${ }^{*} p<0.05,{ }^{* *} p<0.01$ (LSD Method, and data was log transformed prior to analysis). D. H\&E staining (upper panel) and IHC analysis of GRP94 (lower panel) of WT and $c 94^{f f f}$ uteri from 4 and 8 weeks. Scale bar, $50 \mu \mathrm{m}$. White arrows indicate glands and black and red bars denote columnar luminal epithelial cells and SCM, respectively. 
from $c P^{f / f}$ uteri that typically involved the full thickness of the endometrium and abutted the myometrium. All $c P^{f /}$ f94/f uteri showed extensive squamous metaplasia of the endometrial luminal lining (Figure 4E, red bars). At 8 weeks, $c P^{f / f}$ uteri continued to show aggressive EAC, and SCM of the luminal epithelium was detected (Figure 4F). Strikingly, 8 week old $c P^{f f f} 94^{f / f}$ uteri only showed low grade lesions characterized by large well-differentiated glandular structures with no morphological features of invasiveness such as ragged edges or stromal reactions (Figure 4F). GRP94 depletion in both 4 and 8 week $c P^{f / f} 94^{f / f}$ uteri, including in the well-differentiated glandular structures, was confirmed by IHC (Figures 4E and 4F).

\section{$c P^{f / f} 94^{f f f}$ uteri display early squamous metaplasia and reduced $\beta$-catenin signaling}

Through IHC, we observed luminal epithelial cells were positive for both p63 and K14 at 4 week old $c P^{f f f} 94^{f / f}$ uteri (Figure 5A), indicating accelerated onset of SCM compared to age-matched $c 94^{f f f}$ and $c P^{f f f}$ uteri. Active nuclear $\beta$-catenin expression was readily detected in the WT and $c P^{f / f}$ but not the $c P^{f f f} 94^{f / f}$ uteri while total $\beta$-catenin was robustly expressed in all three genotypes (Figure 5B). As a critical transcription factor, nuclear $\beta$-catenin activates downstream targets such as cyclin D1 which induces progression through the G1 phase of the cell cycle [29]. Expression of cyclin D1 was more prominent in the transformed glandular structures in $c P^{f f f}$ uteri compared to WT epithelium attesting to increased cell cycle activity (Figure 5B). Expression of cyclin D1 was low and confined to the basal layers in the metaplastic epithelium of $c P^{f / f} 94^{f / f}$ uteri, indicating an ordered proliferation and a level of organization characteristic of untransformed squamous epithelium (Figure 5B). At 8 weeks, IHC for K14 confirmed that PTEN-null uteri showed SCM in some areas and, interestingly, those same regions showed low GRP94 expression (Figure 5C, broken lines). Thus, both $c 94^{f / f}$ and $c P^{f f f}$ uteri showed squamous differentiation, correlating with GRP94 deficiency. We compared the distribution of metaplastic squamous cells (K14 positive) versus columnar cells (K8 positive) in $\mathrm{cP}^{\mathrm{ff} / \mathrm{f}} 94^{\mathrm{f} / \mathrm{f}}$ uteri. Costaining for K14 (green) and K8 (red) showed that at 4 weeks, whereas endometrial glands were K8 positive (white arrow), most of the endometrial luminal cells expressed the marker of squamous differentiation (K14) with some residual cells located in the top layer (luminal surface) expressing K8
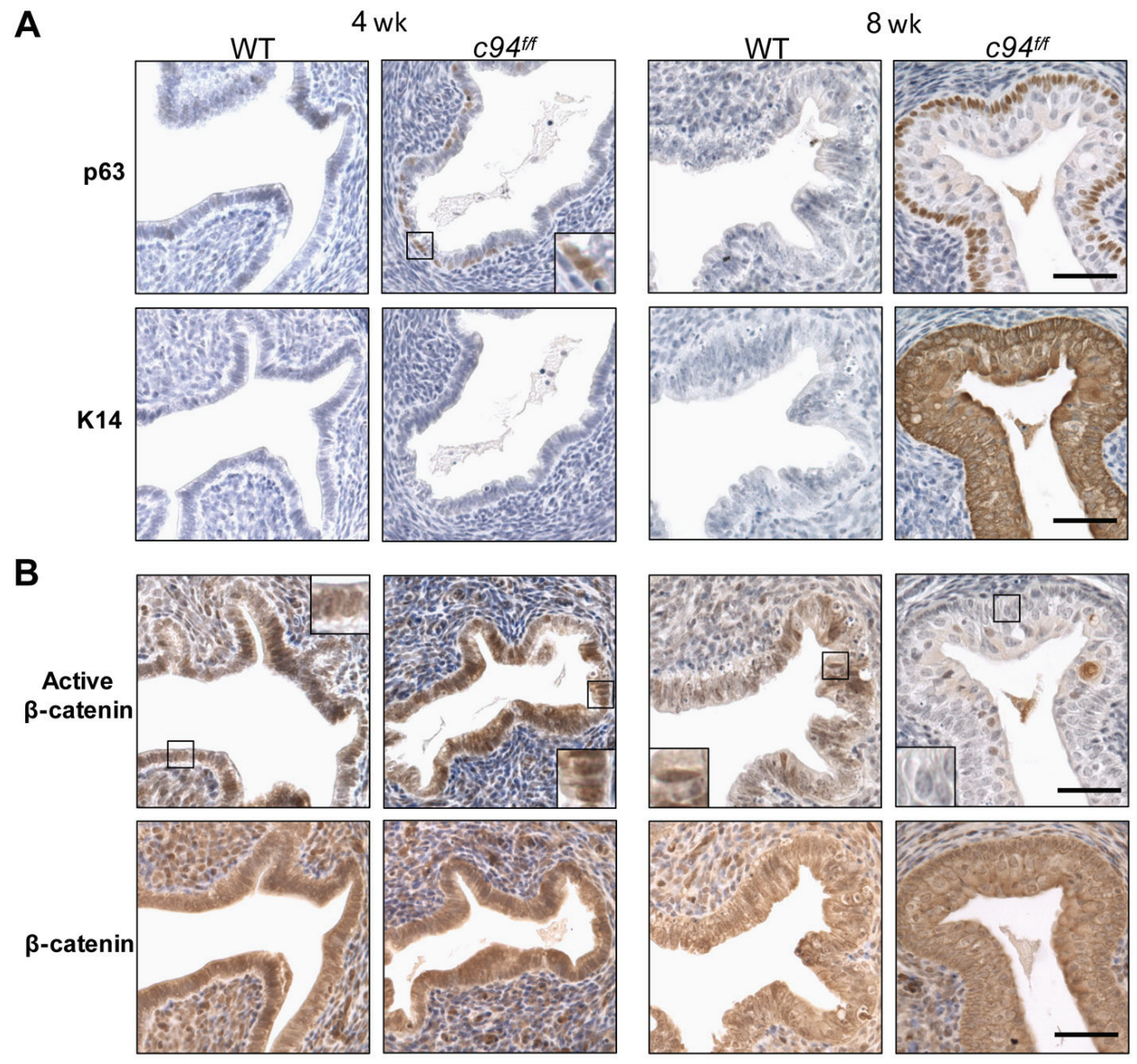

Figure 3: Induction of squamous metaplasia in developing $\mathbf{c} 94^{\mathrm{fff}}$ uteri. A. IHC of p63 and cytokeratin 14 (K14). B. IHC of active $\beta$-catenin and $\beta$-catenin in WT and $c 94^{f f f}$ uteri at 4 and 8 weeks. Enlarged views of the boxed regions are shown. Scale bar, $50 \mu \mathrm{m}$. 
(Figure 5D). By 8 weeks, both endometrial luminal and glandular cells expressed the squamous marker (K14), with some cells expressing glandular marker (K8) located near the glandular lumen. Occasional cells expressed both K14 and K8 markers (Figure 5D, yellow arrows and box). Intraand extra-cellular mucin, indicative of secretory activity, was present in 8 week old $c P^{f f} 94^{f f}$ uteri, as confirmed by PASdiastase (Figure 5D) and mucicarmine (not shown) positivity.

\section{Attenuation of AKT/S6 activation and proliferation in $c P^{f f / 9} 4^{f / f} \mathrm{SCM}$}

Pten deletion leads to PI3K signaling with activation of downstream targets including AKT and S6 via phosphorylation [30]. As expected, $c P^{f f f}$ uteri showed robust activation of S6 in the EAC (Figure 6A, white arrows) and luminal epithelial cells (black bars), whereas in $c P^{f f f} 94^{f f f}$ uteri S6 activation was confined to the glands but absent in the SCM (Figure 6A, red bars). Immunofluorescence (IF) analysis for activated AKT showed a pattern similar to that of pS6 in the three genotypes such that AKT phosphorylation was evident in the glands of $c P^{f f f}$ and $c P^{f f f} 94^{f f f}$ uteri (white arrows), but substantially reduced in the SCM of the $c P^{f f f} 4^{f f f}$ uteri (Figure 6B, red bars) compared to the columnar luminal epithelial cells of $c P^{f f f}$ uteri (white bars). IHC analysis showed no ERK activation in uteri of all three genotypes (Supplementary Figure S3). Proliferation as measured by IHC markers expressed either throughout the cell cycle (Ki67) or during the mitotic phase (phospho-histone H3), was substantially reduced in the $c P^{f f f} 94^{f f f}$ compared to $c P^{f f f}$ uteri and was confined to glandular cells and basal layer of SCM (Figures 6C and 6D). We investigated the potential contribution of apoptosis to the reduced size of $c P^{f f f} 94^{f / f}$ uteri. IHC showed that whereas $c P^{f f}$ uteri exhibited a few cells positive for cleaved caspase-3 located towards the lumen, $c P^{f f f} 94^{f f f}$ uteri showed little if any cells positive for cleaved caspase-3 (Supplementary Figure S4). Collectively, these results indicate that suppression of AKT/S6 activation and reduced proliferation, rather than leading to increased apoptosis, likely contribute to the suppression of PTEN-null driven EAC in $c P^{f f f} 94^{f f}$ uteri.
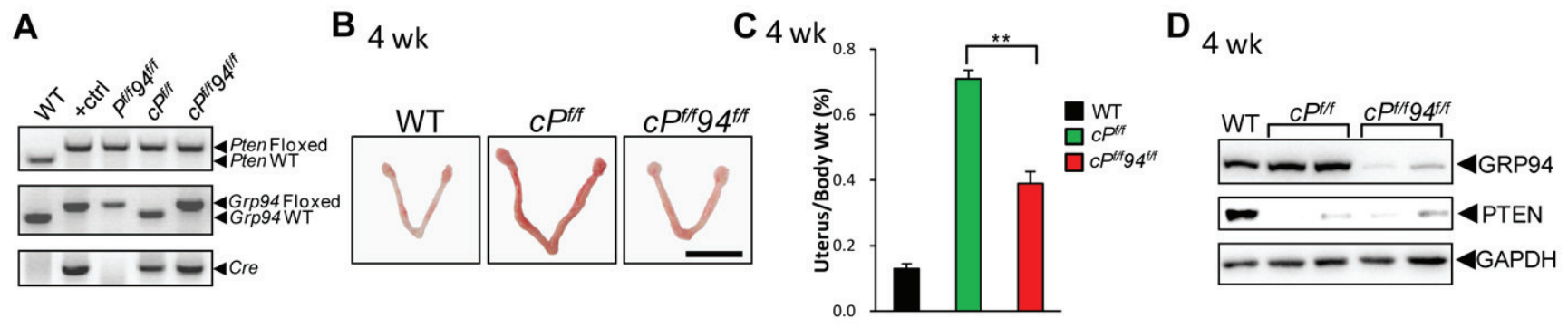

E 4 wk
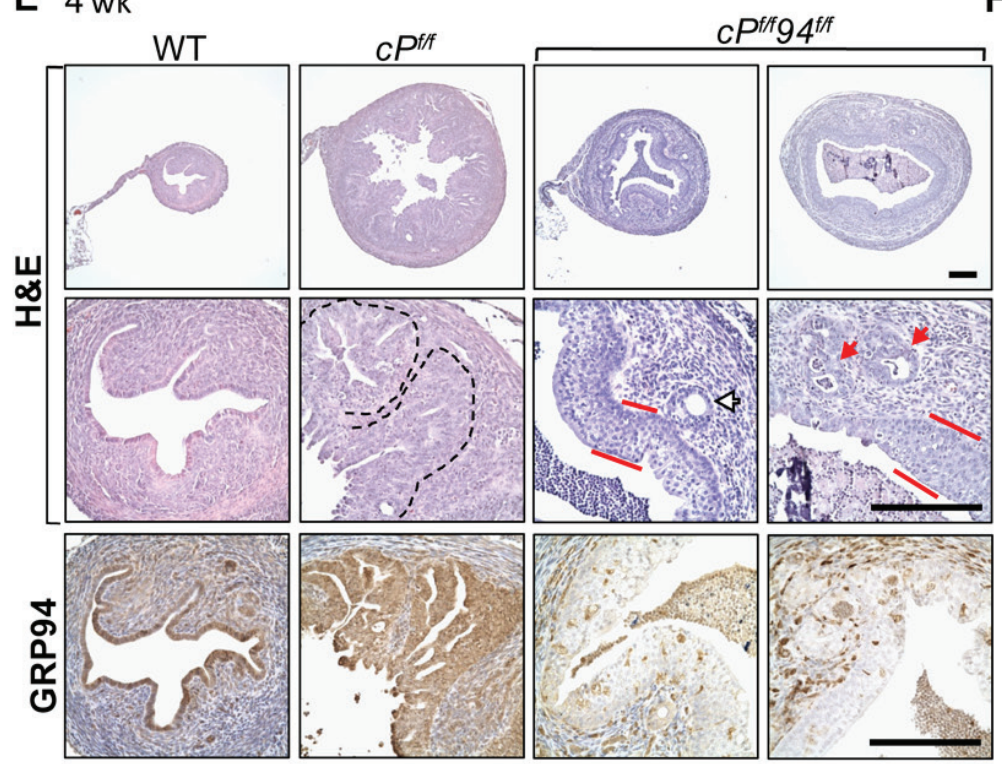

F $8 w k$
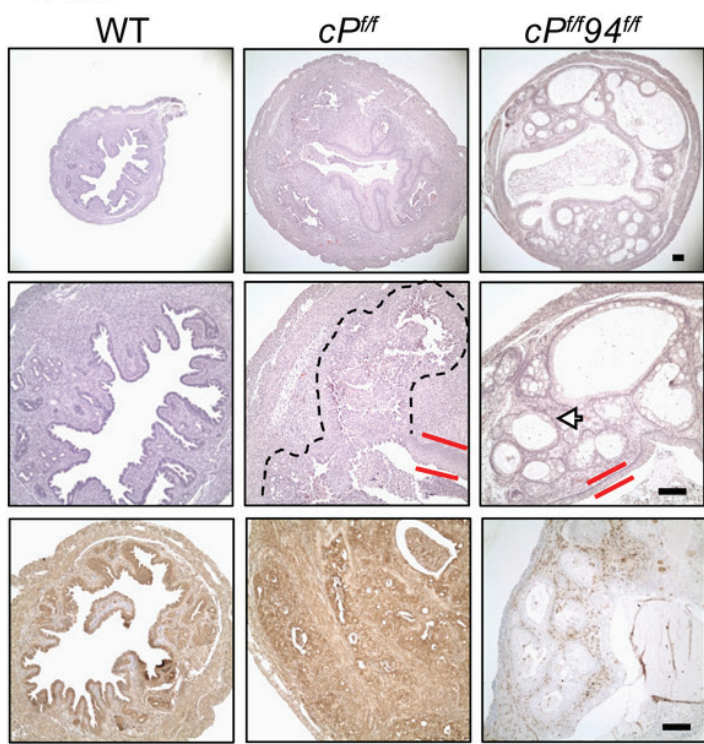

Figure 4: Reduced endometrial cancer in $\boldsymbol{c P}^{\mathrm{f} / f} \mathbf{9} 4^{f / f}$ uteri. A. Representative mouse tail PCR genotyping of the indicated alleles. B. Representative gross anatomy of uteri of the indicated genotype at 4 weeks. Scale bar, $1 \mathrm{~cm}$. C. The ratio of uterine weight to body weight in WT $(\mathrm{n}=16), c P^{f f f}(\mathrm{n}=10)$ and $c P^{f f f} 94^{f f}(\mathrm{n}=9)$ mice at 4 weeks. The data are presented as mean \pm s.e., ${ }^{* *} p<0.01$ (LSD Method). D. Western blot analysis of GRP94 and PTEN levels in uteri of indicated genotypes with GAPDH serving as the loading control. E. H\&E staining and IHC of GRP94 in uteri of the indicated genotype at 4 weeks and F. at 8 weeks. White and red arrows indicate normal and transformed glands, respectively, the broken lines denote EAC and red bars denote SCM. Scale bar, $200 \mu \mathrm{m}$. 


\section{$c^{f f f} 94^{f f f}$ uteri show no EAC myometrial invasion and high E-cadherin expression}

We next compared myometrial invasion of $c P^{f f}$ and $c P^{f f f} 4^{\text {ff }}$ uteri through histological examination of H\&E stained tissue sections by a pathologist (L.D.), as well as examination of IHC stains on consecutive microtome sections for a muscle layer marker, $\alpha$-smooth muscle actin $(\alpha$-SMA) and an epithelial cell marker, pan-cytokeratin (panCK) [3]. Myometrial invasion was readily observed in $c P^{f f f}$ uteri at 8 weeks (Figure 7A, arrows). In contrast, no myometrial invasion was detected in age-matched $c P^{f f f} 94^{f f f}$ uteri (Figure 7A). Seven of $10 c P^{f f f}$ uteri exhibited myometrial invasion at that age compared to 0 out of $8 \mathrm{cPff} g 4^{f f f}$ uteri (Figure 7B). These differences were statistically significant $(p=0.004)$. Consistent with this notion that invasive cancer cells loosen their connection to neighboring cells and basement membrane via downregulation of intercellular adhesion [12], $c P^{f f}$ uteri in which myometrial invasion was present, exhibited very low level of E-cadherin
(Figures 7C and 7D). In contrast, both WT and $c P^{f f f} 94^{f f f}$ uteri showed high E-cadherin expression in epithelial cells.

\section{Prolonged depletion of GRP94 in PTEN-null uteri leads to massive glandular expansion but no EAC formation}

In following WT, $c P^{f f}$ and $c P^{f f f} 94^{f f f}$ mice past 8 weeks, we noticed that the size of the $c P^{f f f} 4^{f f f}$ uteri was considerably larger than the $c P^{f f}$ uteri by 12 and 20 weeks (Supplementary Figure S5A). For both 12 and 20 week uteri, histological examination showed aggressive EAC in the $c P^{f f}$ uteri, whereas the $c P^{f f f} 94^{f f f}$ uteri displayed massively expanded, but mature glands with abundant secretions lacking any of the cytological and architectural features typically associated with malignancy (Figure 7E). IHC further showed that GRP94 depletion had persisted in the $c P^{f f f} 94^{f f f}$ uteri at least through 20 weeks (Supplementary Figure S5B). The effects of GRP94 deficiency in the WT (c94ff) and PTEN-null uteri $\left(c P^{f f} 94^{f f}\right)$ are summarized in Figure 8 , with the striking
A $_{4 \mathrm{wk}}$
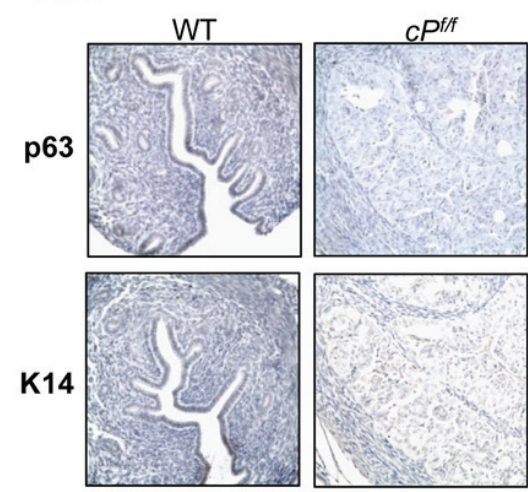

C $8 w k$
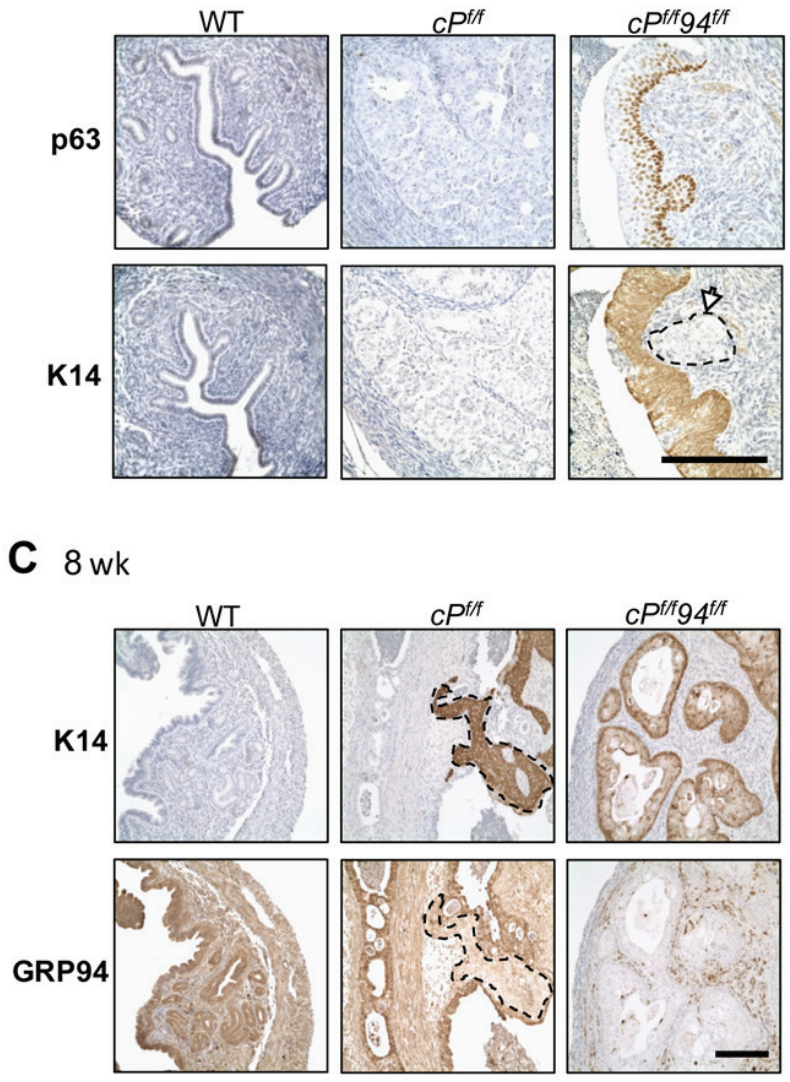

B $4 w k$

$\beta$-catenin
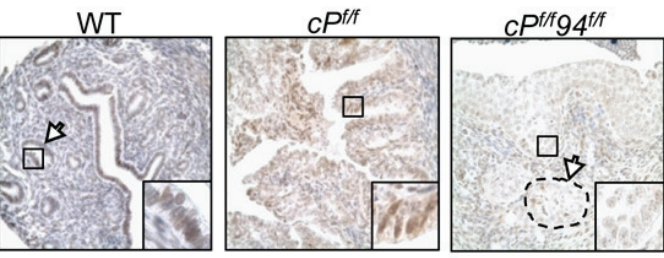

Active

$\beta$-catenin
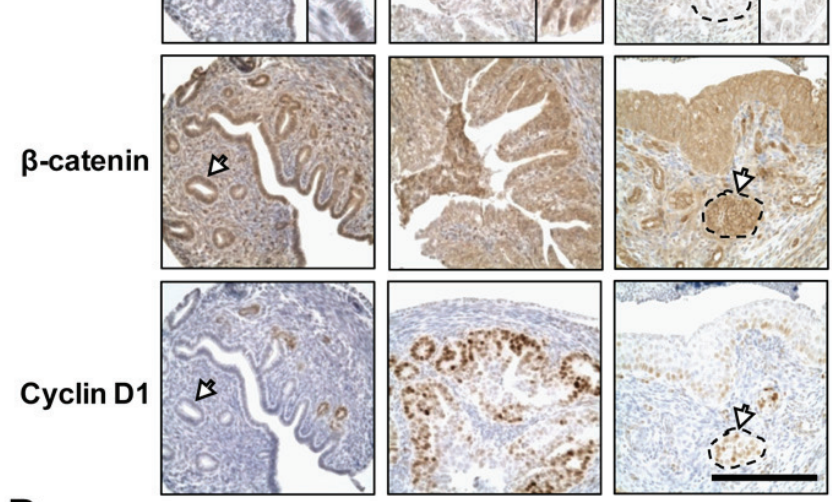

D $_{c P^{f / f} 94^{f / f}}$
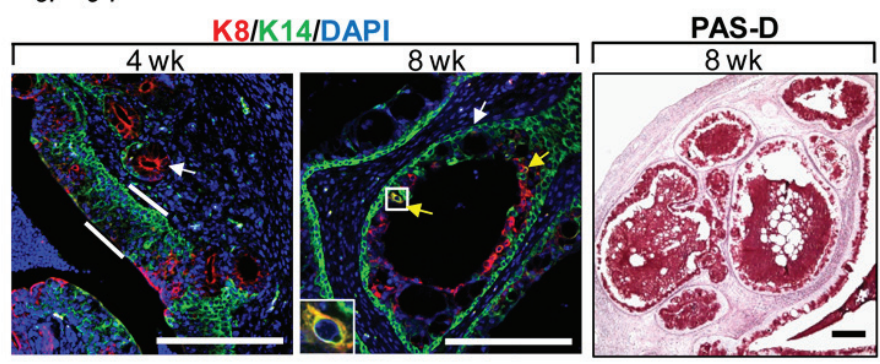

Figure 5: Accelerated squamous metaplasia and reduced $\beta$-catenin signaling in $\boldsymbol{c} \boldsymbol{P P}^{f f f} \mathbf{9} 4^{f f f}$ uteri. A. IHC of p63, K14 and B. IHC of active $\beta$-catenin, $\beta$-catenin, cyclin D1 in uteri of indicated genotypes at 4 weeks. The white arrows denote glands. Enlarged view of the boxed region is shown. C. IHC of K14, GRP94 in uteri of indicated genotypes at 8 weeks. Scale bar, $200 \mu \mathrm{m}$. D. Merged images of immunofluorescence (IF) of K8 (red) and K14 (green) with nuclei stained with DAPI (blue) in $c$ Pfffg fff uteri and PASdiastase staining of $c P^{f f f} q^{f f f}$ uteri at the indicated age. Scale bar, $200 \mu \mathrm{m}$. White arrows indicate glands, white bars denote SCM and yellow arrows indicate cells positive for both K8 and K14. Enlarged view of the boxed region is shown. 
observation that while PTEN-null uteri with WT GRP94 level all developed EAC by 4 weeks, no EAC was detected in the uteri of 12 to 20 week $c P^{f / f} 94^{f / f}$ mice.

\section{DISCUSSION}

The glucose regulated proteins including GRP94 and GRP78 are stress-inducible chaperones that mostly reside in the ER and are well-established as molecular chaperones critical for the ER homeostasis [5]. While GRP94 shares partial amino acid homology with HSP90, its cellular localization and functions are distinct from HSP90 [6]. At the same time while GRP94 and GRP78 are coordinately upregulated by ER stress, knockout of either GRP94 or GRP78 in mouse models resulted in embryonic lethality, implying distinct and non-compensatory functions in early mammalian development $[8,31]$. Recently we have examined the consequence of GRP78 knockout alone or in combination of PTEN in the mouse uteri and discovered that while both GRP78 and GRP94 deficient uteri were smaller in size and showed a decrease in the number of glands, unlike GRP94, GRP78 deficient uteri were lined with a single layer of columnar cells. The uteri from the $c P^{f f f} 78^{f f f}$ uteri showed decreased AKT activation and were devoid of atypical hyperplasia, a well-established EAC precursor, throughout the experimental period of up to 8 months [32].

These interesting observations raise the question whether other ER chaperones such as GRP94 could also impact EAC development, and if so, do they utilize the same or different mechanism? Results from the Cancer
A $4 \mathrm{wk}$
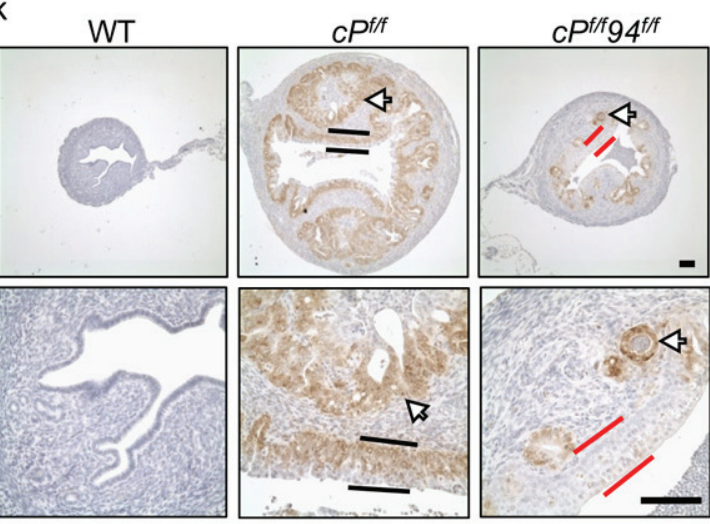

S6
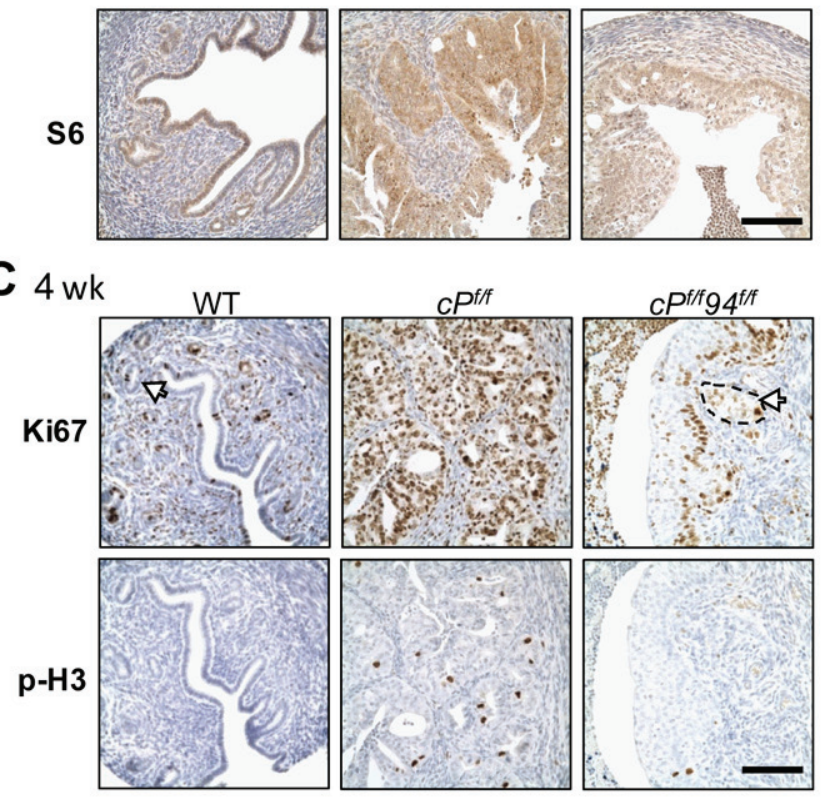

B $4 \mathrm{wk}$

pAKT
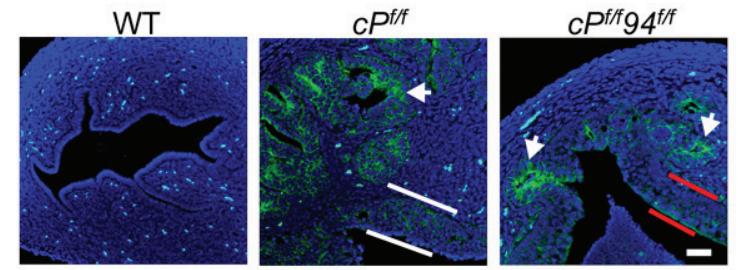

(Ser473)
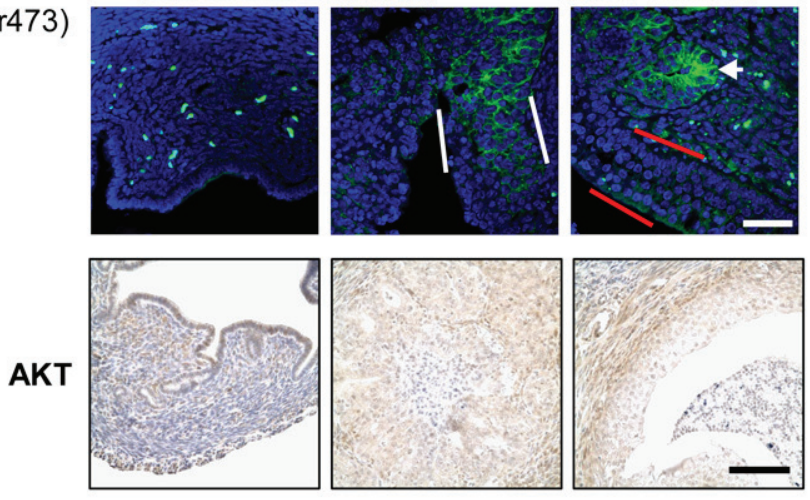

D

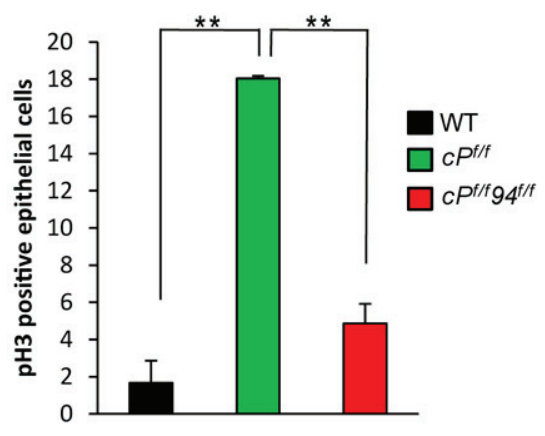

Figure 6: Attenuated AKT activation and decreased proliferation in $\boldsymbol{c P}^{\mathrm{f} / \mathrm{f} / \mathrm{9} / f} \mathbf{S C M}$. A. IHC of phospho-S6 (pS6) and S6 in uteri of the indicated genotypes at 4 weeks. White arrows indicate glands, black and red bars denote columnar luminal epithelial cells and SCM, respectively. B. IF of pAKT (Ser 473) and IHC of AKT. White arrows indicate glands, white and red bars denote columnar luminal epithelial cells and SCM, respectively. C. IHC of Ki67 and phospho-Histone H3 (p-H3) in mouse uteri. Scale bar, $100 \mu \mathrm{m}$. D. Quantification of the $\mathrm{p}-\mathrm{H} 3$ positive epithelial cells in the uteri sections of WT $(n=6), c P^{f f f}(n=3)$ and $c P^{f f f} 4^{f f f}(n=4)$ uteri at 4 weeks. The data are presented as mean \pm s.e., ${ }^{* *} p<0.01$ (LSD Method). 
Genome Atlas analysis in this study provided the first clue that Grp94 mRNA expression is significantly increased in human EAC tissues across all grades of EAC, confirmed by IHC staining of human EAC samples. Recent evidence also showed that GRP94 suppresses AKT activation in hepatocellular carcinoma in vivo and in vitro $[13,33]$, implying that $\mathrm{AKT}$ activation in PTEN-null driven EAC may also be impaired by the loss of GRP94. In this study, we investigated the effect of GRP94 deficiency in the mouse uterus in the absence or presence of the tumor suppressor gene Pten, which is commonly altered in type 1 endometrial cancer. This study reveals new GRP94 function on uteri development and oncogenic signaling which could offer novel mechanisms for curbing EAC.
First, compared to WT, GRP94 deficient uteri exhibited a decrease in the number of endometrial glands, depriving adenocarcinoma of a substrate from which to develop. This could explain in part the lower incidence of EAC in the $c P^{f f f} 94^{f f f}$ uteri, as well as the smaller size of such uteri, since budding of nascent glands from the luminal epithelium is critical for uterine development [34]. Loss of GRP94 might also lead to decreased uterine size through IGF1 deficiency, since GRP94 is an essential chaperone for the production of IGFs [7, 9, 35], and IGF-1 deficient mice possess an infantile uterus with severe hypoplasia especially in the myometrium [36].

Strikingly, uterine GRP94 deficiency resulted in spontaneous replacement of the columnar luminal epithelial cells with squamous cells as early as 8 weeks. Such replacement of one differentiated cell

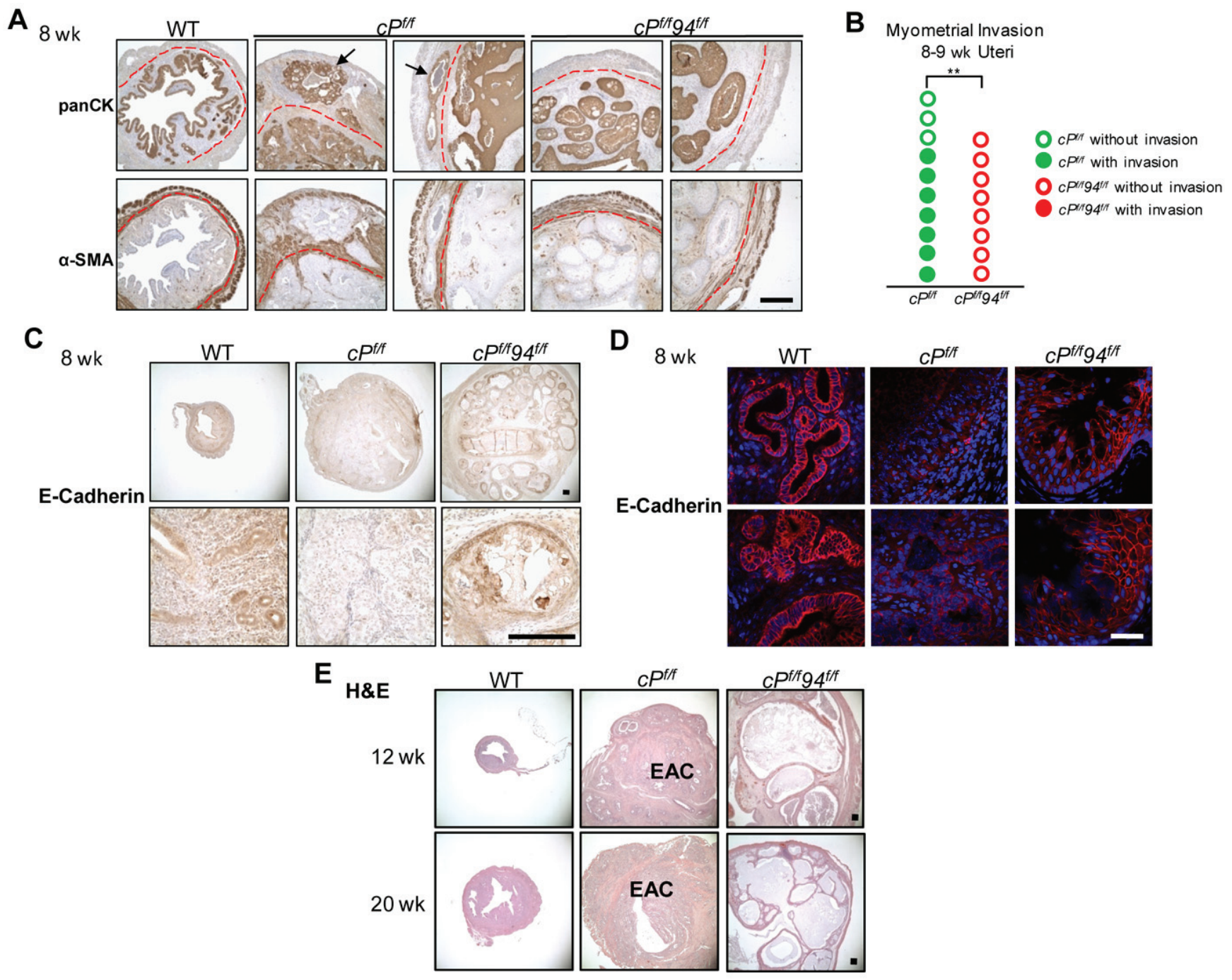

Figure 7: Characterization of $c P^{f f f} 94^{f f f}$ uteri on myometrial invasion and prolonged stage. A. IHC of pan-cytokeratin (panCK) and $\alpha$-smooth muscle actin ( $\alpha$-SMA) on consecutive slides in uteri of the indicated genotypes at 8 weeks. The red broken lines denote the boundaries between the endometrium and the myometrium. Arrows indicate EAC invasion into the myometrium. Scale bar, $400 \mu \mathrm{m}$. B. Frequency of myometrial invasion in $c P^{f f f}$ and $c P^{f f f} 94^{f f f}$ uteri at $8-9$ weeks. Each circle represented one mouse. $p=0.004$ (2-sided Fisher's exact test). C. IHC of E-cadherin at 8 weeks. Scale bar, $200 \mu \mathrm{m}$. D. IF of E-cadherin on frozen tissue sections (red) at 8 weeks. Nuclei were stained with DAPI (blue). Scale bar, $50 \mu \mathrm{m}$. E. H\&E of WT, $c P^{f f f}$ and $c P^{f f f} 94^{f f}$ uteri at 12 weeks (upper panel) and 20 weeks (lower panel). Scale bar, $200 \mu \mathrm{m}$. 
type with another, defined as metaplasia, while a rare occurrence in the healthy endometrium, can be observed occasionally and under both physiological and pathological conditions such as endometrial cancer, chronic irritation or progestin effects [37-39]. The reduced number of glands and the presence of SCM in GRP94-deficient uteri resembled the phenotypes associated with uterine $\beta$-catenin ablation [27]. Indeed, depletion of uterine GRP94 resulted in loss of active nuclear $\beta$-catenin, suggesting that this could contribute in part to spontaneous SCM in the $c 94^{f f f}$ uteri. SCM was accelerated when GRP94 deficiency was superimposed on a PTEN deficiency, which independently induces endometrial SCM [40]. In support of the notion that GRP94 deficiency promotes SCM induction, we observed that the SCM cells in the PTEN-null uteri also expressed low GRP94. Interestingly, uterine depletion of GRP78, in the presence [32] or absence of PTENdeficiency did not induce SCM (Supplementary Figure S1), underscoring the unique role of GRP94 in SCM.

Our work provides the first evidence that GRP94 deficiency can delay the development of cancers driven by the absence of a functional PTEN. Interestingly, this study revealed that the majority of $c P^{f f f} 94^{f / f}$ uteri at 4 weeks did develop endometrial cancer albeit the tumors were less aggressive and much smaller in overall volume compared to the EAC seen in the $c P^{f f f}$ uteri. However, by 8 weeks, the morphological appearance of the $c P^{f f f} 94^{f f f}$ tumors had become substantially different than that of the tumors seen in the $c P^{f / f}$ uteri. The lesions were low-grade and less aggressive as evidenced by smooth acinar contours and abundant secretions indicative of higher degree of differentiation. The $c P^{f f f} 94^{f / f}$ uteri showed WT level of E-cadherin and no sign of myometrial invasion by tumor. We further noted that in 8 week $c P^{f f f} 94^{f / f}$ uteri, the majority of the cells expressed the squamous marker (K14). Cells expressing glandular marker (K8) were located in the luminal layer, suggesting that the precursor cells normally giving rise to columnar epithelial cells alternatively initiate a different differentiation pathway leading to squamous cells. Beyond 8 weeks, the $c P^{f f f} 94^{f f}$ uteri rapidly increased in size and the endometrium showed expanded glands with abundant secretions. The accumulation of secretion could be due either to an effect of GRP94 deficiency on secretory activity or to entrapment of secretions within glandular lumina because of narrowing of secretory ducts due to squamous metaplasia. In summary, loss of uterine GRP94 leads to several unique phenotypes clearly distinguishable from those associated with suppression of EAC development in conditional GRP78 knockout mice [32]. We show that replacing columnar epithelial cells with squamous epithelial cells induced by GRP94 deficiency may represent a novel mechanism for suppression of EAC.
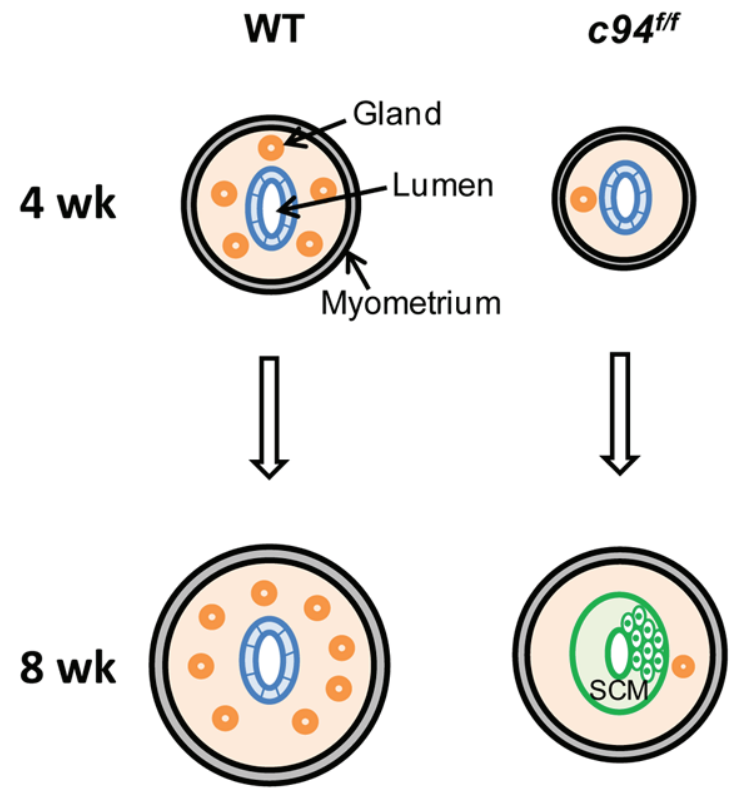

Active $\beta$-catenin $\downarrow$

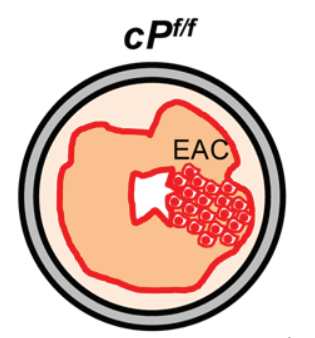

Active $\beta$-catenin $\uparrow$ Cyclin D14 p-AKT, p-S64

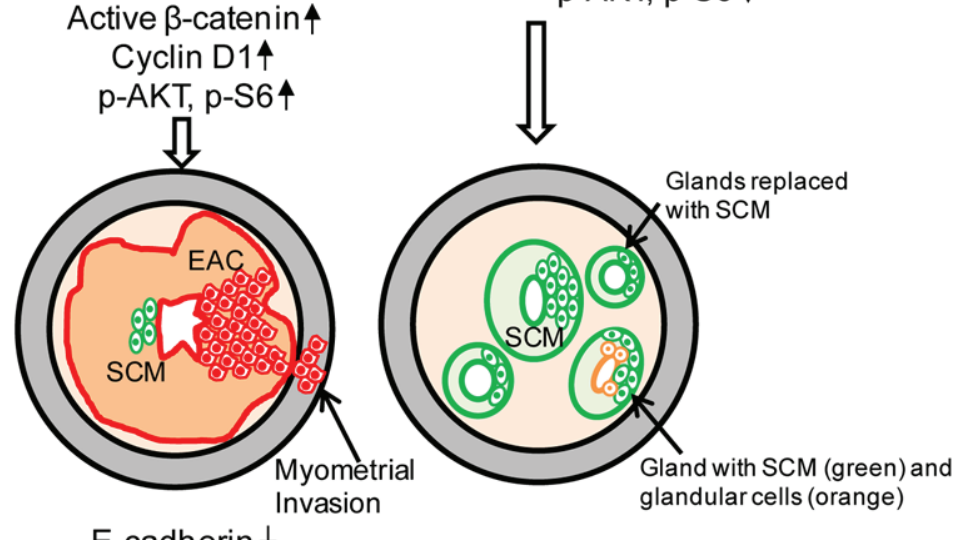

E-cadherin $\downarrow$

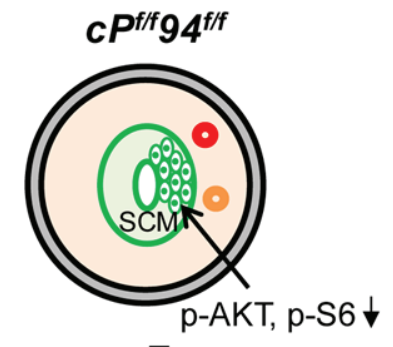

glandular cells (orange)

Figure 8: Summary model on the effect of GRP94 deficiency in mouse uteri in the presence or absence of PTENdeficiency. The development of 4 and 8 week uteri of the indicated genotypes is shown. The morphological transitions of the glands, SCM replacement, EAC formation, alteration of the signaling pathways and E-cadherin expression are summarized for 4 and 8 week uteri. EAC: endometrial cancer. SCM: squamous cell metaplasia. 


\section{MATERIALS AND METHODS}

Mice

PR-Cre;Pten fff mice on a C57BL6/129SV background [3] were crossed with ten $^{\text {fff }}$;Grp $94^{f / f}$ mice [13] to generate the PR-Cre;Pten ${ }^{f / f} ; G r p 94^{f / f}$ ( $\left(P^{f / f} 94^{f / f}\right.$ ) mice. Grp $94^{\text {fff }}$ mice on a C57BL/6;129/SV background [8] were crossed with $c P^{f / f 9} 94^{f / f}$ to generate PR-Cre;Grp $94^{f / f}$ $\left(c 94^{f f}\right)$. Genotyping was performed by PCR using mouse tail genomic DNA as previously described [13]. All protocols for animal use and euthanasia were reviewed and approved by the University of Southern California Institutional Animal Care and Use Committee.

\section{Tissue processing and histology}

Female mice were euthanized and uteri were isolated. Collected samples were either frozen in liquid nitrogen for biochemical analysis or fixed in $10 \%$ zinc formalin (Sigma-Aldrich, St. Louis, MO) or frozen in OCT compound (Tissue-Tek Sakura, Torrance, CA) for tissue analysis. Both paraffin-embedded and OCTembedded tissues were sectioned at $7 \mu \mathrm{m}$.

\section{Western blot analysis}

Tissue lysates were subjected to SDS-PAGE and Western blot analysis as described previously [41]. Primary antibodies used were GRP94 (1:5000, Enzo Life Sciences, Farmingdale, NY), PTEN (1:1000, Cell Signaling, Danver, MA) and GAPDH (1:5000, Santa Cruz Biotechnology, Dallas, TX).

\section{Tissue section staining}

Immunostaining on paraffin-embedded or frozen tissue sections was performed as described previously $[13,42]$. Tissue sections were incubated at $4^{\circ} \mathrm{C}$ overnight with primary antibodies against GRP94 (1:250, Enzo Life Sciences, Farmingdale, NY), cytokeratin 14 (1:400), p63 (1:200), Cyclin D1 (1:100), Ki67 (1:200) from Thermo Scientific (Fremont, CA), $\beta$-catenin (1:100, Santa Cruz Biotechnology, Dallas, TX), active $\beta$-catenin $(1: 50$, Millipore, Billerica, MA), phospho-S6 (1:200), S6 (1:50), phospho-AKT (Ser 473, 1:50), AKT (1:200), phosphoHistone H3 (1:200) from Cell Signaling Technology (Danver, MA), cytokeratin 8 (1:100, Developmental Studies Hybridoma Bank, Iowa City, IA), $\alpha$-Smooth Muscle Actin (1:2000, Sigma-Aldrich, St. Louis, MO), pan cytokeratin (1:50, Abcam, Cambridge, MA) and E-cadherin (1:50, BD Biosciences, San Jose, CA). Immunofluorescence was analyzed using a Zeiss LSM 510 confocal microscope with LSM 510 Version 4.2 SP1 acquisition software. Confocal images were acquired with $20 \mathrm{X}$ or $40 \mathrm{X}$ oil lens. Images were then processed with LSM Image Browser R4.2 and Adobe Photoshop CS5.
Staining for mucin by either mucicarmine or periodic acid-Schiff stain (PAS) followed by diastase digestion was performed by the clinical histology laboratory at University of Southern California using standard protocols.

\section{Cell lines and culture conditions}

Human endometrial cancer cell lines AN3CA and ECC1 have been described and authenticated by mitochondrial sequencing (7.1.11) [23]. Only short term cultures from the verified frozen stocks were used. The cells were maintained in Dulbecco's Modified Eagle media (Corning, Manassas, VA) supplemented with $10 \%$ fetal bovine serum (Omega Scientific, Tarzana, CA), $1 \%$ penicillin and streptomycin at $37^{\circ} \mathrm{C}$ in a humidified atmosphere of $5 \% \mathrm{CO}_{2}$.

\section{WST-1 viability assay}

Cell viability was assessed with the WST-1 reagent (Roche, Indianapolis, IN). Briefly, 10,000 cells per well were plated onto 96-well plates and the cell viability was measured by incubating each plate with $10 \mu \mathrm{l} /$ well of WST-1 substrate for 2 hours and plates were red at a wavelength of $450 \mathrm{~nm}$.

\section{RNA-seq dataset analyses}

TCGA Level 3 RNA-seq data were downloaded from TCGA data access (https://tcga-data.nci.nih.gov/ tcgafiles/ftp_auth/distro_ftpusers/anonymous/tumor/). The version number of RNA-seq dataset is IlluminaHiSeq RNASeqV2_3.1.12. The data were all generated on Illumina HiSeq platform, mapped with the RSEM algorithm and normalized so that the third quartile for each sample equals 1000. Entrez gene IDs were used for mapping to genomic locations using GenomicRanges [43].

To compare the mRNA expression level of Grp94 and Pten between normal and endometrial cancer samples, we $\log 2$ transformed the expression data $[\log 2(\mathrm{RSEM}+1)]$, and then performed a Student's $t$-test on gene expression between 24 normal and 177 tumor samples. In order to investigate the gene expression correlation between Grp94 and Pten, Pearson correlation test was used on $\log 2$ transformed expression data.

\section{Statistical analysis}

Statistical analysis was performed with the least significant difference (LSD) method, the 2-tailed Fisher's exact test or 2-tailed Student's $t$-test as indicated.

\section{ACKNOWLEDGMENTS}

We thank Paulette Mhawech-Fauceglia for pathology consulting, Susan Groshen for assistance in biostatistical analysis, Agnieszka Kobielak, Wei Li, WanTing Chen, Chun-Chih Tseng, Dat Ha, Nancy Wu and 
members of the Lee lab for providing reagents, assistance and helpful discussions.

\section{CONFLICTS OF INTEREST}

The authors declare no conflict of interest.

\section{GRANT SUPPORT}

This work was supported by National Institute of Health grants R01 CA027607 (A.S. Lee), R01 CA133117 (L. Dubeau) and K08 CA175161 and Stop Cancer Career Development award (Y.G. Lin). The USC Norris Comprehensive Cancer Center Transgenic Core and Biostatistics Core were supported by NCI grant P30 CA014089. The Cell and Tissue Imaging Core of the USC Research Center for Liver Diseases was supported by NIH grant P30 DK048522.

\section{Editorial note}

This paper has been accepted based in part on peerreview conducted by another journal and the authors' response and revisions as well as expedited peer-review in Oncotarget.

\section{REFERENCES}

1. Siegel RL, Miller KD and Jemal A. Cancer statistics, 2015. CA Cancer J Clin. 2015; 65:5-29.

2. Di Cristofano A and Ellenson LH. Endometrial carcinoma. Annu Rev Pathol. 2007; 2:57-85.

3. Daikoku T, Hirota Y, Tranguch S, Joshi AR, DeMayo FJ, Lydon JP, Ellenson LH and Dey SK. Conditional loss of uterine Pten unfailingly and rapidly induces endometrial cancer in mice. Cancer Res. 2008; 68:5619-5627.

4. Ni $\mathrm{M}$ and Lee AS. ER chaperones in mammalian development and human diseases. FEBS Lett. 2007; 581:3641-3651.

5. Lee AS. Glucose-regulated proteins in cancer: molecular mechanisms and therapeutic potential. Nat Rev Cancer. 2014; 14:263-276.

6. Luo B and Lee AS. The critical roles of endoplasmic reticulum chaperones and unfolded protein response in tumorigenesis and anticancer therapies. Oncogene. 2013; $32: 805-818$.

7. Marzec M, Eletto D and Argon Y. GRP94: An HSP90-like protein specialized for protein folding and quality control in the endoplasmic reticulum. Biochim Biophys Acta. 2012; 1823:774-787.

8. Mao C, Wang M, Luo B, Wey S, Dong D, Wesselschmidt $\mathrm{R}$, Rawlings $\mathrm{S}$ and Lee AS. Targeted mutation of the mouse Grp94 gene disrupts development and perturbs endoplasmic reticulum stress signaling. PLoS One. 2010; 5:e10852.

9. Wanderling S, Simen BB, Ostrovsky O, Ahmed NT, Vogen SM, Gidalevitz T and Argon Y. GRP94 is essential for mesoderm induction and muscle development because it regulates insulin-like growth factor secretion. Mol Biol Cell. 2007; 18:3764-3775.

10. Liu B, Yang Y, Qiu Z, Staron M, Hong F, Li Y, Wu S, Li Y, Hao B, Bona R, Han D and Li Z. Folding of Toll-like receptors by the HSP90 paralogue gp96 requires a substrate-specific cochaperone. Nat Commun. 2010; 1:79.

11. Liu B, Staron M, Hong F, Wu BX, Sun S, Morales C, Crosson CE, Tomlinson S, Kim I, Wu D and Li Z. Essential roles of grp94 in gut homeostasis via chaperoning canonical Wnt pathway. Proc Natl Acad Sci USA. 2013; 110:6877-6882.

12. Gupta GP and Massague J. Cancer metastasis: building a framework. Cell. 2006; 127:679-695.

13. Chen WT, Tseng CC, Pfaffenbach K, Kanel G, Luo B, Stiles BL and Lee AS. Liver-specific knockout of GRP94 in mice disrupts cell adhesion, activates liver progenitor cells, and accelerates liver tumorigenesis. Hepatology. 2014; 59:947-957.

14. Luo B, Lam BS, Lee SH, Wey S, Zhou H, Wang M, Chen SY, Adams GB and Lee AS. The endoplasmic reticulum chaperone protein GRP94 is required for maintaining hematopoietic stem cell interactions with the adult bone marrow niche. PLoS One. 2011; 6:e20364.

15. Hong F, Liu B, Chiosis G, Gewirth DT and Li Z. alpha7 helix region of alphaI domain is crucial for integrin binding to endoplasmic reticulum chaperone gp96: a potential therapeutic target for cancer metastasis. J Biol Chem. 2013; 288:18243-18248.

16. Reddy RK, Dubeau L, Kleiner H, Parr T, Nichols P, Ko B, Dong D, Ko H, Mao C, DiGiovanni J and Lee AS. Cancerinducible transgene expression by the Grp94 promoter: spontaneous activation in tumors of various origins and cancer-associated macrophages. Cancer Res. 2002; 62:7207-7212.

17. Hua Y, White-Gilbertson S, Kellner J, Rachidi S, Usmani SZ, Chiosis G, Depinho R, Li Z and Liu B. Molecular chaperone gp96 is a novel therapeutic target of multiple myeloma. Clin Cancer Res. 2013; 19:6242-6251.

18. Morales C, Rachidi S, Hong F, Sun S, Ouyang X, Wallace C, Zhang Y, Garret-Mayer E, Wu J, Liu B and Li Z. Immune chaperone gp96 drives the contributions of macrophages to inflammatory colon tumorigenesis. Cancer Res. 2014; 74:446-459.

19. Chen WT, Ha D, Kanel G and Lee AS. Targeted deletion of ER chaperone GRP94 in the liver results in injury, repopulation of GRP94-positive hepatocytes, and spontaneous hepatocellular carcinoma development in aged mice. Neoplasia. 2014; 16:617-626.

20. Rachidi S, Sun S, Wu BX, Jones E, Drake RR, Ogretmen B, Cowart LA, Clarke CJ, Hannun YA, Chiosis G, Liu B and Li Z. Endoplasmic reticulum heat shock protein gp96 maintains liver homeostasis and promotes hepatocellular carcinogenesis. J Hepatol. 2015; 62:879-888. 
21. Cancer Genome Atlas Research Network, Kandoth C, Schultz N, Cherniack AD, Akbani R, Liu Y, Shen H, Robertson AG, Pashtan I, Shen R, Benz CC, Yau C, Laird PW, et al. Integrated genomic characterization of endometrial carcinoma. Nature. 2013; 497:67-73.

22. Djordjevic B, Hennessy BT, Li J, Barkoh BA, Luthra R, Mills GB and Broaddus RR. Clinical assessment of PTEN loss in endometrial carcinoma: immunohistochemistry outperforms gene sequencing. Mod Pathol. 2012; 25:699-708.

23. Gray MJ, Mhawech-Fauceglia P, Yoo E, Yang W, Wu E, Lee AS and Lin YG. AKT inhibition mitigates GRP78 (glucose-regulated protein) expression and contribution to chemoresistance in endometrial cancers. Int J Cancer. 2013; 133:21-30.

24. Soyal SM, Mukherjee A, Lee KY, Li J, Li H, DeMayo FJ and Lydon JP. Cre-mediated recombination in cell lineages that express the progesterone receptor. Genesis. 2005; 41:58-66.

25. Yang A, Kaghad M, Wang Y, Gillett E, Fleming MD, Dotsch V, Andrews NC, Caput D and McKeon F. p63, a p53 homolog at 3q27-29, encodes multiple products with transactivating, death-inducing, and dominant-negative activities. Mol Cell. 1998; 2:305-316.

26. Koster MI and Roop DR. Mechanisms regulating epithelial stratification. Annu Rev Cell Dev Biol. 2007; 23:93-113.

27. Jeong JW, Lee HS, Franco HL, Broaddus RR, Taketo MM, Tsai SY, Lydon JP and DeMayo FJ. beta-catenin mediates glandular formation and dysregulation of betacatenin induces hyperplasia formation in the murine uterus. Oncogene. 2009; 28:31-40.

28. Villacorte M, Suzuki K, Hirasawa A, Ohkawa Y, Suyama M, Maruyama T, Aoki D, Ogino Y, Miyagawa S, Terabayashi T, Tomooka Y, Nakagata N and Yamada G. beta-Catenin signaling regulates Foxa2 expression during endometrial hyperplasia formation. Oncogene. 2013; 32:3477-3482.

29. Shtutman M, Zhurinsky J, Simcha I, Albanese C, D'Amico $\mathrm{M}$, Pestell R and Ben-Ze'ev A. The cyclin D1 gene is a target of the beta-catenin/LEF-1 pathway. Proc Natl Acad Sci USA. 1999; 96:5522-5527.

30. Song MS, Salmena L and Pandolfi PP. The functions and regulation of the PTEN tumour suppressor. Nat Rev Mol Cell Biol. 2012; 13:283-296.

31. Luo S, Mao C, Lee B and Lee AS. GRP78/BiP is required for cell proliferation and protecting the inner cell mass from apoptosis during early mouse embryonic development. Mol Cell Biol. 2006; 26:5688-5697.

32. Lin YG, Shen J, Yoo E, Liu R, Yen HY, Mehta A, Rajaei A, Yang W, Mhawech-Fauceglia P, DeMayo FJ, Lydon J, Gill
$\mathrm{P}$ and Lee AS. Targeting the glucose-regulated protein-78 abrogates Pten-null driven AKT activation and endometrioid tumorigenesis. Oncogene. 2015; 34:5418-5426.

33. Huang CY, Batzorig U, Cheng WL, Huang MT, Chen WY, Wei PL and Chang YJ. Glucose-regulated protein 94 mediates cancer progression via AKT and eNOS in hepatocellular carcinoma. Tumour Biol. 2016; 37:4295-4304.

34. Cooke PS, Spencer TE, Bartol FF and Hayashi K. Uterine glands: development, function and experimental model systems. Mol Hum Reprod. 2013; 19:547-558.

35. Barton ER, Park S, James JK, Makarewich CA, Philippou A, Eletto D, Lei H, Brisson B, Ostrovsky O, Li Z and Argon Y. Deletion of muscle GRP94 impairs both muscle and body growth by inhibiting local IGF production. FASEB J. 2012; 26:3691-3702.

36. Baker J, Hardy MP, Zhou J, Bondy C, Lupu F, Bellve AR and Efstratiadis A. Effects of an Igfl gene null mutation on mouse reproduction. Mol Endocrinol. 1996; 10:903-918.

37. Anderson MC, Robboy SJ and Russell P. Endometritis, metaplasia, polyp and miscellaneous changes. Robboy SJ, Mutter GL, Prat J, Bentley R, Russell P, Anderson MC, editors. London: Churchill Livingstone; 2002.

38. Miranda MC and Mazur MT. Endometrial squamous metaplasia. An unusual response to progestin therapy of hyperplasia. Arch Pathol Lab Med. 1995; 119:458-460.

39. Lee YB, Kim CS, Ro JY, Kwak HM and Song CH. Long term effects of IUD on the human endometrium. Histologic, histochemical and ultrastructural studies. Yonsei Med J. $1983 ; 24: 141-148$.

40. van der Zee M, Jia Y, Wang Y, Heijmans-Antonissen C, Ewing PC, Franken P, DeMayo FJ, Lydon JP, Burger CW, Fodde R and Blok LJ. Alterations in Wnt-beta-catenin and Pten signalling play distinct roles in endometrial cancer initiation and progression. J Pathol. 2013; 230:48-58.

41. Ye R, Jung DY, Jun JY, Li J, Luo S, Ko HJ, Kim JK and Lee AS. Grp78 heterozygosity promotes adaptive unfolded protein response and attenuates diet-induced obesity and insulin resistance. Diabetes. 2010; 59:6-16.

42. Fu Y, Wey S, Wang M, Ye R, Liao CP, Roy-Burman $\mathrm{P}$ and Lee AS. Pten null prostate tumorigenesis and AKT activation are blocked by targeted knockout of ER chaperone GRP78/BiP in prostate epithelium. Proc Natl Acad Sci USA. 2008; 105:19444-19449.

43. Lawrence M, Huber W, Pages H, Aboyoun P, Carlson M, Gentleman R, Morgan MT and Carey VJ. Software for computing and annotating genomic ranges. PLoS Comput Biol. 2013; 9:e1003118. 